\title{
In-cylinder soot precursor growth in a low-temperature combustion diesel engine: laser-induced fluorescence of polycyclic aromatic hydrocarbons
}

\author{
C.A.J. Leermakers \\ Department of Mechanical Engineering, Eindhoven University of Technology, P.O. Box 513, GEM-N 1.21, 5600 MB, Eindhoven, The Netherlands
}

\author{
M.P.B. Musculus*
}

Combustion Research Facility, Sandia National Laboratories, P.O. Box 969, MS 9053, Livermore, CA 94551, USA

*Corresponding author: Fax: +1 (925) 294-1004, e-mail: mpmuscu@sandia.gov

Colloquium: IC engine and gas turbine combustion (alternatives: Soot, $\mathrm{PAH}$, and other large molecules; Diagnostics)

Word count according to method 1:

\begin{tabular}{|c|c|c|c|c|c|}
\hline Introduction & & & 409 & 411 & \\
\hline Body & & & 2763 & 2894 & \\
\hline Conclusions & & & 454 & 456 & \\
\hline Acknowledgements & & & & 66 & \\
\hline Equations & & & & $\mathbf{0}$ & \\
\hline Nomenclature & & & & $\mathbf{0}$ & \\
\hline References & 21 & 24 & 402 & 454 & 4281 \\
\hline Tables & lines & cols & & & 4094 \\
\hline 1 & 13 & 1 & & 114 & 114 \\
\hline Figures & h (mm) & cols & caption & & \\
\hline 1 & 80 & 1 & 25 & 223 & \\
\hline 2 & 109,3 & 2 & 69 & 593,92 & \\
\hline 3 & 65,9 & 2 & 72 & 405,96 & \\
\hline 4 & 50,8 & 1 & 35 & 168,76 & \\
\hline 5 & 49,5 & 1 & 33 & 163,9 & \\
\hline & & & & & 1556 \\
\hline & & & 5764 & 5951 & \\
\hline
\end{tabular}

The authors DO NOT agree to pay charges for color reproduction of figures. All figures to be printed in grey scale.

\begin{abstract}
The growth of poly-cyclic aromatic hydrocarbon (PAH) soot precursors are observed using a two-laser technique combining laser-induced fluorescence (LIF) of PAH with laser-induced incandescence (LII) of soot in a diesel engine under low-temperature combustion (LTC) conditions. The broad mixture distributions and slowed chemical kinetics of LTC “stretch out” soot-formation processes in both space and time, thereby facilitating their study. Imaging PAH-LIF from pulsed-laser excitation at three discrete wavelengths (266, 532, and $633 \mathrm{~nm}$ ) reveals the temporal growth of PAH molecules, while soot-LII from a 1064-nm pulsed laser indicates inception to soot. The distribution of PAH-LIF also grows spatially within the combustion chamber before soot-LII is first detected. The PAH-LIF signals have broad spectra, much like LII, but typically with spectral profile that is inconsistent with laser-heated soot. Quantitative naturalemission spectroscopy also shows a broad emission spectrum, presumably from PAH chemiluminescence, temporally coinciding with of the PAH-LIF.
\end{abstract}

Keywords: Polycyclic aromatic hydrocarbons; Particulate matter; Laser-induced fluorescence; Laser-induced incandescence; Diesel low-temperature combustion 


\section{Introduction}

Strategies to reduce pollutant emissions from diesel engines while maintaining fuel efficiency are of interest for meeting environmental regulations and market demands. Low-temperature combustion (LTC) is a class of in-cylinder strategies that uses dilute mixtures and enhanced premixing to maintain or even improve fuel efficiency while reducing pollutant emissions, including soot [1]. In addition to its practical utility, the broad mixture distributions and slowed chemicalkinetics of LTC conditions "stretch out" soot-formation processes in both space and time, thereby facilitating their study.

Poly-cyclic aromatic hydrocarbons (PAH) are an important class of soot precursors [2]. Smaller PAH with one to five rings may be either fuel-borne [3] or combustion-generated. Larger $\mathrm{PAH}$ with more than five rings are almost entirely due to combustion-induced formation and/or growth. In addition to toxicity/carcinogenicity [4], PAH formation is undesirable because PAH contribute to particulate matter (soot) [5].

PAH growth during combustion can be observed to some degree through spectroscopic techniques. The absorption/emission spectra of PAH molecules shift to longer wavelengths with increasing number of rings [6,7]. Individual PAH spectra are broadband and overlap with each other, however, so specific PAH species generally cannot be isolated spectrally.

One technique to probe the absorption redshift due to PAH growth is laser-induced fluorescence (LIF). If soot is present, the laser-induced emission can also include significant laser-induced incandescence (LII) of soot, which is also spectrally broad. One way to discriminate soot-LII from PAH-LIF is to use a second laser pulse at a wavelength that yields only soot-LII. The Nd:YAG laser fundamental at $1064 \mathrm{~nm}$ should yield only soot-LII, because even very large PAH do not display single-photon absorption at near-infrared wavelengths [8,9]. Comparisons of the two signals, acquired virtually simultaneously, thus allow discrimination of PAH-only regions from regions with both PAH and soot.

This two-laser LIF-LII approach for diesel combustion follows previous work [10], using only 532-nm PAH-LIF. Here, the PAH-LIF is extended over a broader excitation wavelength range, adding 266 and $633 \mathrm{~nm}$, to study the spatiotemporal evolution of both small and very large PAH. Excitation at $266 \mathrm{~nm}$ probes small PAH that do not absorb at 532 $\mathrm{nm}$, while very large PAH could conceivably absorb at $633 \mathrm{~nm}$ and thus fluoresce upon 633-nm excitation. Such absorption, if present, would confound soot extinction techniques that use 633-nm (i.e. HeNe) laser light [11]. Both the soot-LII and the PAH-LIF signals are also spectrally resolved to further discriminate LIF from LII, and quantitative chemiluminescence spectra provide further insight into PAH growth.

\section{Experiment set-up}

\subsection{Optical engine and operating conditions}

The optical engine is a single-cylinder Bowditch-piston version of a Cummins N14 heavy-duty diesel (bore 139.7 mm, stroke $152.4 \mathrm{~mm}, 2.34 \mathrm{~L}$ ). Table 1 shows operating conditions and some engine specifications (see [12,13] for more engine details).

In the experiment schematic of Fig. 1, a flat UV-grade fused-silica piston-crown window provides imaging access from below to the open, right-cylindrical bowl (diameter $97.8 \mathrm{~mm}$ ). A 30-mm-wide curved window matching the piston bowlwall contour and a flat rectangular cylinder-wall window provide laser access into the piston bowl, even near top-dead center.

A target load of 6 bar gross indicated mean-effective pressure (gIMEP) is investigated at 1200 rpm with a single fuel injection per cycle. The fuel is n-heptane, selected for its low fluorescence compared to pump diesel fuel to avoid interference with the PAH-LIF and soot-LII signals. It also has essentially zero fuel-borne PAH, meaning all PAH in this study is combustion-generated. The ignition properties of n-heptane are similar to European diesel fuel, with a cetane number between 52.5 and 56 [14]. Different intake oxygen mole fractions (not including fuel) were achieved by diluting the intake stream with nitrogen. An oxygen mole-fraction of $18 \%$ mimics typical current diesel combustion using exhaust aftertreatment [15], while $10.5 \%$ intake oxygen is low enough that exhaust aftertreatment for soot and nitrogen oxides (NOx) may not be required [15]. Additionally, the low combustion temperatures at 10.5\% oxygen further slow PAH/soot kinetics so that their formation pathways can be studied in more detail. 


\subsection{Excitation setup}

Two independent 10-Hz Spectra Physics Quanta Ray Nd:YAG laser systems provide the two excitation beams. One (model GCR-250) is used at its fundamental $(1064 \mathrm{~nm}$ ) for soot-LII. A second (PRO-200) excites PAH-LIF using either its second $(532 \mathrm{~nm})$ or fourth $(266 \mathrm{~nm})$ harmonics. Additionally, a Spectra-Physics optical parametric oscillator provides pulsed laser light at $633 \mathrm{~nm}$.

One at a time, the 266/532/633-nm laser beams are combined with the 1064-nm beam and co-aligned with the approximate symmetry plane of one of the eight fuel jets, angled at $14^{\circ}$ relative to the firedeck $\left(152^{\circ}\right.$ included angle). The beams are formed into $30-\mathrm{mm}$ wide sheets that are less than one mm thick over the region of interest with pulse energies entering the engine of 50-60 mJ at 266 and $532 \mathrm{~nm}, 40 \mathrm{~mJ}$ at $633 \mathrm{~nm}$, and $250 \mathrm{~mJ}$ at $1064 \mathrm{~nm}$. The effect of divergence through the curved window on laser fluence is minimal within the LII plateau. The 1064-nm fluence exceeds $0.8 \mathrm{~J} / \mathrm{cm}^{2}$, which was found to be sufficient to reach the plateau in the LII signal strength, such that a further increase in fluence does not significantly affect the LII signal. In lower-pressure flames, at the beginning of the observed plateau soot vaporization is occurring but not excessive. The laser fluence in UV and visible is significantly lower than for IR (as can be determined from the ratio of total laser energies, since the beam footprints are similar).

\subsection{Detector setup}

As shown in Fig. 1, the soot-LII signal is directed by the 45-degree mirror and a 50-50 beam splitter to a Xybion gated, intensified CCD camera (model ISQ-350-W-3). The camera is equipped with SWP450 (450-nm short-wave-pass) and GG385 (385-nm long-pass) filters and a 105-mm focal-length glass lens at f/4. As described in [16], laser-heated (LII) soot particles should follow Planck's law and therefore emit more strongly at shorter wavelengths than combustionheated soot, so using a shorter spectral band from 385 to $450 \mathrm{~nm}$ improves the signal-to-noise ratio relative to combustion-heated soot. The laser pulse is bracketed with the minimum intensifier gate width of $60 \mathrm{~ns}$.

The PAH-LIF signal is imaged by a PI-MAX3 ICCD camera with an HQf intensifier and another 105-mm glass lens at $\mathrm{f} / 4$. The intensifier gate overlaps the laser pulse with the minimum achievable 2.47 -ns width to maximize the signal-tonoise ratio. A short-wave-pass filter with a 50\% cut-off near $850 \mathrm{~nm}$ (CVI/Melles-Griot FSWP-850) removes scattered 1064-nm light. Narrow-band notch filters and a WG285 color filter reject elastically scattered light at 532/633 and 266 $\mathrm{nm}$, respectively.

In each engine run, 20 images are acquired at a fixed crank angle, with every fourth image capturing background luminosity without any laser light. The LII laser pulse trails the LIF pulse by $1 \mu \mathrm{s}$, which is sufficient to separate the two signals while being virtually simultaneous relative to engine time scales. The order of the two pulses did not affect the acquired images, as observed previously [10]. From each image set, a single representative image pair is objectively identified using a statistical image-correlation technique [17].

For spectral measurements, an Oriel 77250 monochromator is used as a spectrograph by mounting a Xybion intensified CCD camera (model ISG-250-UX-3) at the exit plane. A 300 lines/mm grating (Oriel \#77939) resolves the 400 to 700 $\mathrm{nm}$ range with a spectral resolution of $8 \mathrm{~nm}$ at $500 \mathrm{~nm}$. The entrance slit is lens-coupled to the probe volume using a 50$\mathrm{mm}$ glass lens at $\mathrm{f} / 4$ (Fig. 1). Spectrograph images are acquired in sets of 20 at each crank angle. For laser-induced spectra, the minimum intensifier gate of $110 \mathrm{~ns}$ brackets the desired laser pulse, whereas for chemiluminescence spectra the gate is increased to 140 microseconds, or about $1^{\circ} \mathrm{CA}$. The laser-induced spectra are background-luminositysubtracted (removing very weak natural emission), median-filtered, spatially binned over the head of the jet (35-47mm from the injector along the nominal jet axis), and ensemble-averaged. The chemiluminescence spectra are only spatiallyand ensemble-averaged. All spectra are made quantitative in spectral radiance by correcting for the filter transmission and camera spectral sensitivity using a Hoffman calibrated integrating sphere, model LR-6Z.

Finally, a large-area $\left(1 \mathrm{~cm}^{2}\right)$ silicon, photoconductive mode photodiode (UDT PIN-10D) viewing through the pistoncrown window records the natural combustion-luminosity, with system sensitivity set to resolve the strong soot luminosity, but not the relatively weak chemiluminescence. 


\section{Results and discussion}

Figure 2 shows image sets for 10.5\% and 18\% intake-oxygen, with 532-nm LIF and 1064-nm LII acquired virtually simultaneously in each image, but each image-pair at a different crank angle is from a different engine run. LIF and LII images are false-colored green and red, respectively, such that overlap regions appear yellow. To improve the yellow visibility relative to green (which appears very bright to the eye), the gamma [18] of the red image is set to 0.8. Because of the possibility of soot-LII in the signal from 532-nm (as well as 266- and 632-nm) excitation, overlap areas may or may not have PAH, but certainly have soot. The injector position and bowl-rim position are indicated in the images, with one of the eight fuel jets propagating horizontally left to right across the image.

Because of the different dynamic ranges of the cameras and to make initial soot-formation visible, both the LII and the LIF signal images are artificially amplified such that 0.1 percent of the pixels in the area of interest saturates. These postprocessing gains, color-coded according to the false-color scheme, are displayed in each image. The left side of each image in Fig. 2 shows various degrees of scattering/interference from the 1064-nm beam (red). The scattering appears worse in the early images because of higher gains (as indicated on the images).

\subsection{2-nm LIF and 1064-nm soot LII imaging at 10.5\% and 18\% intake oxygen}

The top of Fig. 2 shows the measured injection rate, the apparent rate of heat release (ROHR, from an air-standard firstlaw analysis [19]) and the soot-luminosity photodiode signal. Vertical dashed-lines indicate the crank angles of the images. The ROHR and soot luminosity show that ignition and soot-formation processes are slower at $10.5 \%$ intake oxygen than at $18 \%$ oxygen.

With 10.5\% intake oxygen, 532-nm PAH-LIF (green) is first detected immediately after the premixed heat-release peak, near 370 CAD. Thereafter, the PAH-LIF area grows without any detectable soot-LII until 374 CAD, at which time the photodiode also detects soot natural luminosity (top). After this $4{ }^{\circ} \mathrm{CA}$ inception delay, soot-LII signal quickly fills the regions where $\mathrm{PAH}$ were present, yielding increasing signal on both cameras (note decreasing gains in later images).

Also for 10.5\% intake oxygen, a conventional combusting diesel jet structure [16] is not apparent because of the long mixing time. Instead, the soot distribution is a consequence of jet/wall/swirl dynamics preceding soot formation. Prior to ignition and combustion, the jet impinges and spreads along the bowl wall. The counterclockwise swirl-flow sweeps the originally horizontal jet partially out of the laser sheet, while parts of the adjacent clockwise jet are transported into the bottom of the laser sheet. Hence, the large structure in the bottom of the images at the end of the sequence (378 CAD) is a remnant of the interaction between two adjacent jets.

For 18\% intake oxygen (Fig. 2, right), a more conventional combusting diesel jet is apparent. Over the narrower crankangle range of images (to $366 \mathrm{CAD}$ ) than at 10.5\% oxygen (to $378 \mathrm{CAD}$ ), the jet is transported counterclockwise only slightly by swirl. PAH-LIF is again first detected immediately after the premixed burn (361 CAD). Within $1{ }^{\circ} \mathrm{CA}$, the PAH-LIF is typically surrounded by soot-LII. While some portions display pure PAH (green), the rapid increase in soot (note the decreasing 'red' gains) yields LII signal on both cameras. Soot luminosity is also much higher than at $10.5 \%$ intake oxygen (Fig. 2, top).

\subsection{Multi-wavelength imaging comparison}

LIF originating from 266, 532, and 633-nm excitation is compared at 10.5\% intake oxygen fraction, where the evolution of PAH is slowest and thus most easily studied. Fig. 3 shows three time-sequences of representative composite images from LIF excitation at these three wavelengths (false-colored green) with simultaneous 1064-nm LII (false-colored red).

Comparing the left and middle sets of images in Fig. 3 shows that combustion-generated species, very likely PAH, can be detected earlier with 266-nm than with 532-nm excitation. The onset of fluorescence resulting from excitation at 266 $\mathrm{nm}$ occurs between 368 and 369 CAD. In the 370 and 371 CAD images, the signal for 266-nm excitation is stronger than for 532-nm excitation. Recall that the images use different gains as indicated on each image.

Excitation at $633 \mathrm{~nm}$ shows signal only at later crank angles, starting from 371 CAD. Furthermore, signal from 633-nm excitation, presumably fluorescence of very large PAH, arises before any LII. This implies that (very large) PAH can absorb light at the red HeNe-laser wavelength $(\sim 633 \mathrm{~nm})$. Similar long-wavelength PAH absorption/fluorescence has also been observed in atmospheric-pressure flames, even to $660 \mathrm{~nm}$ [21]. While this to some extent may open 
possibilities for fluorescence detection of such species, absorption by very large PAH may also interfere with sootextinction measurements that use HeNe lasers; the extinction will be increased beyond that caused by soot alone, especially near soot inception. At later crank angles when soot is more dense, absorption by PAH would likely become less significant, or even insignificant, relative to extinction by soot.

All three image sequences show a consistent PAH signal development. A temporal redshift in absorption (excitation) wavelength, and hence growth in PAH molecular size (i.e., number of rings), occurs over a short $3-4{ }^{\circ} \mathrm{CA}$ range. In the ensemble, no spatial differences are apparent among the different excitation-wavelength/size classes; in regions where long-wavelength LIF is observed, similar regions also show short-wavelength LIF (though from different cycles).

Figure 3 shows that to detect the earliest (and smallest) PAH, 266-nm laser light is appropriate. However, laser-light as well as the corresponding emission are more readily attenuated at shorter wavelengths. While most of the windowdeposited soot in the path of the laser is blasted away by laser pulses between skip-fired cycles, attenuation by the soot cloud itself [20] and soot deposits on the piston window can be problematic, especially at high-sooting conditions. Furthermore, components of commercially available fuels, as well as (intermediate) combustion products, may be excited by 266-nm laser light, thereby confounding the fluorescence signal. Hence, while 266-nm PAH-LIF can be useful for fuels/conditions such as those employed here, excitation of PAH-LIF at $532 \mathrm{~nm}$ has a broader utility.

\subsection{Spectral analysis of LIF and LII at $10.5 \%$ intake oxygen}

Figure 4 shows quantitative LIF and LII spectra for the 10.5\% oxygen condition of Fig. 3. Spectral ranges where the filters have high absorbance are artificially masked for clarity. At 374 CAD, at which time the soot-LII (not shown) is relatively weak, the 532-nm LIF spectrum is broad, peaking near $550 \mathrm{~nm}$, and without any narrow peaks attributable to small combustion intermediates such as $\mathrm{CH}$ or $\mathrm{C}_{2}$. Shortly thereafter, at $376 \mathrm{CAD}$, the broad 532-nm LIF emission is more redshifted, peaking near $600 \mathrm{~nm}$. The 1064-nm soot-LII spectrum at 376 CAD is similarly broad, but exhibiting an even more redshifted peak between 600 and $650 \mathrm{~nm}$. As explained earlier, the emission from 532-nm excitation likely includes soot LII when soot is present, so the similarity to the soot-LII spectrum from 1064-nm excitation is not unexpected. At even later timings (not shown), the LIF spectra become even more similar to the soot-LII spectra, suggesting that the signal from 532-nm excitation becomes dominated by soot-LII.

The 266 and 633-nm LIF emissions are also spectrally resolved at the same two crank angles as for $532 \mathrm{~nm}$. Considering 266-nm excitation first, at the early crank angle of 374 CAD, the LIF spectrum is much different than the LII signal, peaking at $<450 \mathrm{~nm}$, which is inconsistent with blackbody incandescence at the soot vaporization temperature (peaking at 640-720 nm at 4000-4500 K). (The peak may actually lie in the ultraviolet, which is not resolved in these measurements.) Hence, emission from 266-nm excitation at this and earlier crank angles can be interpreted as being almost solely LIF, not having significant LII, consistent with the images in Fig. 3. At later crank angles, the signal likely contains more and more LII, resulting in a red-shift of the spectral peak.

The 633-nm induced LIF spectra are somewhat weaker than for 266 and 532-nm excitation (see Fig. 3), especially at 374 CAD. Nonetheless, the spectrum at 374 CAD displays proportionally more light red-shifted from the 633-nm excitation wavelength than those of the shorter wavelength excitations. At the later crank angle (376 CAD) the shape of the LII and 633-nm LIF spectra are so similar that no LIF signal can be distinguished from LII.

A significant portion of the early LIF emission (at 374 CAD) for both 532-nm and 633-nm excitation is blue-shifted from the laser wavelength. (A similar effect may occur for 266-nm, but is not resolved here). A similar blueshift has been observed in both atmospheric pressure premixed methane and diesel-spray diffusion flames [21], as well as a previous study with this same engine under conditions with no detectable 1064-nm soot LII signal [10]. The source of the blueshifted emission is likely laser-excitation of PAH [21]. We speculate that combustion chemistry yields PAH in excited ro-vibrational states, which can relax to lower ground states after electronic excitation by the laser light, thereby yielding blue-shifted emission. For large PAH molecules with so many ro-vibrational modes, much of the ground electronic state can be in higher ro-vibrational states even for thermal distributions at combustion-heated temperatures. Non-thermal rovibrational distributions resulting from combustion chemistry can increase the amount of blue-shifted emission. Chemiluminescence spectroscopy described in the next section provides some support for the possibility of non-thermal ro-vibrational distributions. 
Figure 5 shows natural chemiluminescence emission spectra for the 10.5\% oxygen condition, using a much longer (140 microsecond) camera gate than the PAH-LIF experiments (110 ns). The spectra span from first heat release to the last crank angle before soot-LII and soot luminosity appear. From the earliest crank angle, the emission spectrum is broadband, peaking in the blue. This early broadband emission spectrum is consistent with the $\mathrm{CO}_{2}{ }^{*}$ emission [22] spectrum, ranging from 340 to about $650 \mathrm{~nm}$ with its peak around $400 \mathrm{~nm}$. Over the next few ${ }^{\circ} \mathrm{CA}$, the broadband signal strength increases, especially at longer wavelengths. A slight hump is visible near $431 \mathrm{~nm}$, corresponding to the excited $\mathrm{CH}^{*}$ radical [23], and a distinct chemiluminescence peak near $590 \mathrm{~nm}$ may be from sodium impurities in the fuel, as sodium-doped flames have a strong isolated line near $589 \mathrm{~nm}$ [22].

The chemiluminescence spectrum departs from the initial $\mathrm{CO}_{2} *$ emission spectrum starting at $368 \mathrm{CAD}$, which is the same crank angle as the first detectable PAH-LIF in the images of Fig. 3 for 266-nm excitation (small PAH). After this initial rise in natural emission (potentially PAH chemiluminescence), its red-peaked broadband emission rises through the same crank-angle range in Fig. 3 where PAH-LIF signals arise with 532-nm (370 CAD) and then 633-nm (371 CAD) excitation. At later crank angles, the natural emission grows rapidly, especially after 374 CAD when soot luminosity and soot-LII become significant in Figs. 2 and 3, respectively. For clarity, the very bright spectra from these later crank angles are not shown here. As the soot luminosity signal eventually becomes orders of magnitude stronger than the chemiluminescence, any narrow spectral features disappear into a broad continuum whose spectral peak shifts further towards the infrared. However, the detection of significant PAH chemiluminescence (molecules in excited electronic states), make it reasonable to expect that also PAH molecules are in excited vibrational states, which could cause the PAH-LIF blue-shift in Fig. 4. Furthermore, the increased short-wavelength emission from PAH chemiluminescence would also explain the unreasonably high apparent soot temperatures observed near the onset of soot formation in twocolor pyrometry measurements, especially for LTC conditions [24].

\section{Summary and Conclusions}

In an optically accessible diesel engine, imaging PAH-LIF from pulsed-laser excitation at 266, 532, and $633 \mathrm{~nm}$ reveals the temporal growth of PAH molecules, while soot-LII from a 1064-nm pulsed laser indicates inception to soot. At a LTC engine operating condition with 10.5\% intake-oxygen, PAH is first detected by $266-\mathrm{nm}$ excitation just after the premixed heat release peak, followed by 532-nm and then 633-nm excitation, indicating a likely temporal growth of PAH molecular size. The PAH-LIF region also grows spatially during the approximately 5-6 ${ }^{\circ} \mathrm{CA}$ before soot-LII is first detected. For $18 \%$ intake oxygen, first PAH are also detected just after the premixed burn, but within $1{ }^{\circ} \mathrm{CA}$, the $\mathrm{PAH}$ region is surrounded by soot, though some images display regions of pure PAH.

The LIF signals have broadband spectra, much like LII, but typically with a blue-shifted peak, such that the LIF spectrum is spectrally inconsistent with laser-heated soot. A substantial portion of the LIF emission is at wavelengths shorter than the excitation laser, suggesting that many of the combustion-generated PAH are in excited ro-vibrational states. The LIF signal with 633-nm excitation is weaker than for 266 or 532-nm excitation, and more difficult to distinguish spectrally from LII because the broadband peaks are at similar wavelengths. Imaging comparisons with 1064-nm soot-LII show that 633-nm LIF does precede LII, however. At later crank angles, the contribution of LII grows to dominate the LIF signals, so that the emission is spectrally indistinguishable from LII.

Quantitative chemiluminescence spectroscopy also shows CH $(430 \mathrm{~nm})$ and sodium $(590 \mathrm{~nm})$ features on a broad emission spectrum, probably from PAH chemiluminescence, whose initial emergence temporally coincides with first detection of the 266-nm PAH-LIF. The existence electronically excited PAH yielding chemiluminescence is consistent with the hypothesis that the strong short-wavelength portion of the LIF emission spectrum is from ro-vibrationally excited PAH, and may also explain anomalously high apparent temperatures at soot inception in soot optical thermometry measurements. At later crank angles, a soot-luminosity signal appears, coinciding with the first soot-LII, and the narrow spectral features disappear as the LII quickly strengthens by orders of magnitude and the spectral peak shifts further toward the infrared.

The detection of apparent PAH-LIF from 633-nm excitation suggest that very large PAH absorb light at HeNe-laser wavelengths in a narrow crank-angle range prior to the formation of soot. While this to some extent may open possibilities for fluorescence detection of such species, absorption by large PAH may also interfere with soot extinction measurements that use HeNe lasers by increasing the extinction beyond that caused by soot alone, especially near soot 
inception. At later crank angles when soot is more dense, absorption by PAH would become less significant, or even insignificant, relative to extinction by soot.

\section{Acknowledgements}

Support for this research at the Combustion Research Facility, Sandia National Laboratories, Livermore, CA, was provided by the U.S. Department of Energy, Office of Vehicle Technologies. Sandia is a multi-program laboratory operated by Sandia Corporation, a Lockheed Martin Company for the United States Department of Energy's National Nuclear Security Administration under contract DE-AC04-94AL85000. Keith Penney and David Cicone are gratefully acknowledged for their assistance with experiments.

\section{References}

[1] M. Musculus, P. Miles, L. Pickett, Prog Energy Combust Sci 39 (2-3) (2013) 246-283.

[2] J. Warnatz, U. Maas, R. Dibble, Combustion, Springer, Berlin-Heidelberg, Germany, 2006.

[3] R.N. Westerholm, T.E. Alsberg, A.B. Fromelln, M.E. Strandell, U. Rannug, L. Winquist, V. Grigoriadis, K.-E. Egebäck, Environmental science \& technology 22 (8) (1988) 925-930.

[4] D.W. Dockery, C. A. Pope, X. Xu, J. D. Spengler, J. H. Ware, M. E. Fay, B. G. Ferris, F. E. Speizer, N Engl J Med 329 (1993) 1753-1759.

[5] H. Bockhorn, Ed., Soot formation in combustion, Springer, Berlin-Heidelberg, 1994.

[6] T. Aizawa, H. Kosaka, Int J Engine Res 9 (2006) 79-96.

[7] K. Hayashida, K. Amagai, K. Satoh, M. Arai, J Eng Gas Turbines Power 128 (2006) 241-246.

[8] R. Rieger, K. Müllen, J Phys Org Chem 23 (2010) 315-325.

[9] R.L. Vander Wal, K.A. Jensen, M.Y. Choi, Combust Flame 109 (1997) 399-414.

[10] M.K. Bobba, M.P.B. Musculus, Combust Flame 159 (2012) 832-843.

[11] M. Musculus, L. Pickett, Combust Flame 141 (4) (2005) 371-391.

[12] J. O'Connor, M. Musculus, SAE Int J Engines 6 (1) (2013) 379-399 (2013-01-0910).

[13] J. O'Connor, M. Musculus, Int. J Eng Research (in press) (2013), doi:10.1177/1468087413488767 .

[14] M. Murphy, J. Taylor, R. McCormick, Compendium of experimental cetane number data, National Renewable Energy Laboratory, NREL/SR-540-36805, 2004.

[15]P. Brijesh, S. Sreedhara, Int J Automotive Technology 14 (2) (2003) 195-206.

[16] J.E. Dec, SAE Trans 106 (3) (1997) 1319-1348 (970873).

[17]C. Genzale, Optimizing Combustion Chamber Design for Low-Temperature Diesel Combustion, PhD thesis, University of Wisconsin-Madison, USA, 2008.

[18] A. Yadav, P. Yadav, Digital image processing, University Science Press, New Delhi, 2009.

[19] J. Heywood, Internal combustion engine fundamentals, McGraw-Hill, 1988.

[20] M.Y. Choi, K.A. Jensen, Combust Flame 112 (4) (1998) 485-491.

[21] S. Bejaoui, R. Lemaire, P. Desgroux, E. Therssen, Appl. Phys. B (in press), 2014.

[22] A.G. Gaydon, The spectroscopy of flames, Chapman and Hall, London, UK, 1974.

[23] Y. Hardalupas, M. Orain, Combust Flame 139 (3) (2004) 188-207.

[24]E. Huestis, P.A. Erickson, M.P.B. Musculus, SAE Trans 116 (4) (2007) 860-879 (2007-01-4017). 
Table 1 Engine specifications and operating conditions

\begin{tabular}{ll}
\hline Compression Ratio & $11.22: 1$ \\
Common-rail injector & Cummins XPI \\
Nozzle hole arrangement & $8 \times 140 \mu \mathrm{m} \times 14^{\circ}$ \\
Fuel rail pressure & $1000 \mathrm{bar}$ \\
Command start of injection & $352 \mathrm{CAD}$ \\
Command injection duration & $2.5 \mathrm{~ms}$ \\
TDC motored density & $16.6 \mathrm{~kg} / \mathrm{m} 3$ \\
TDC motored temperature & $975 \mathrm{~K}$ \\
Intake pressure & $177 \mathrm{kPa}(\mathrm{abs})$ \\
Intake temperature & $155^{\circ} \mathrm{C}$ \\
(Simulated 16:1 intake pressure) & $116 \mathrm{kPa}(\mathrm{abs})$ \\
(Simulated EGR rates) & $30-59 \%$ \\
Air excess ratio & $2.39 \mathrm{at} 21 \% \mathrm{O} 2$ \\
\hline
\end{tabular}

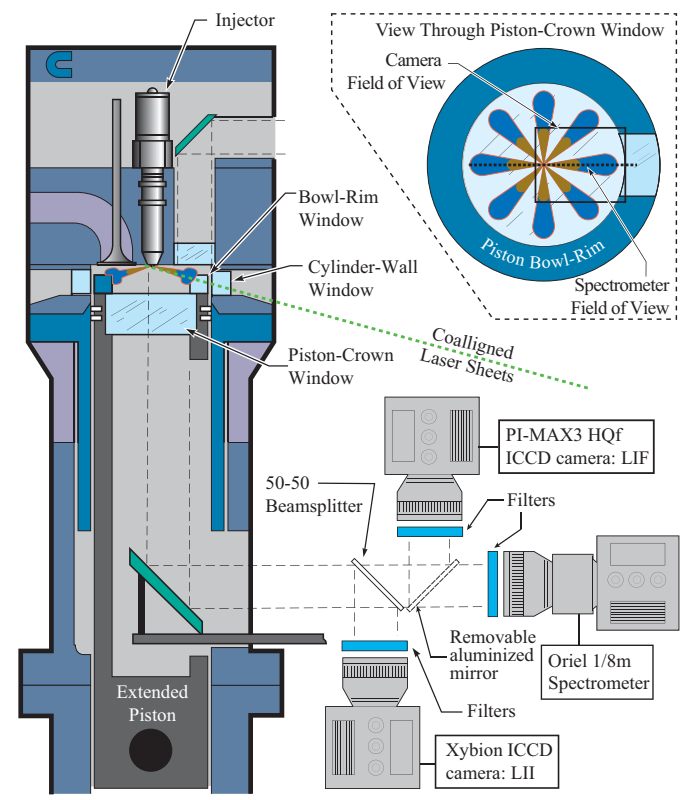

Fig. 1. Schematic of the single-cylinder engine, laser configuration, and optical detector system. The camera and spectrometer field-of-view are shown in the upper right corner. 


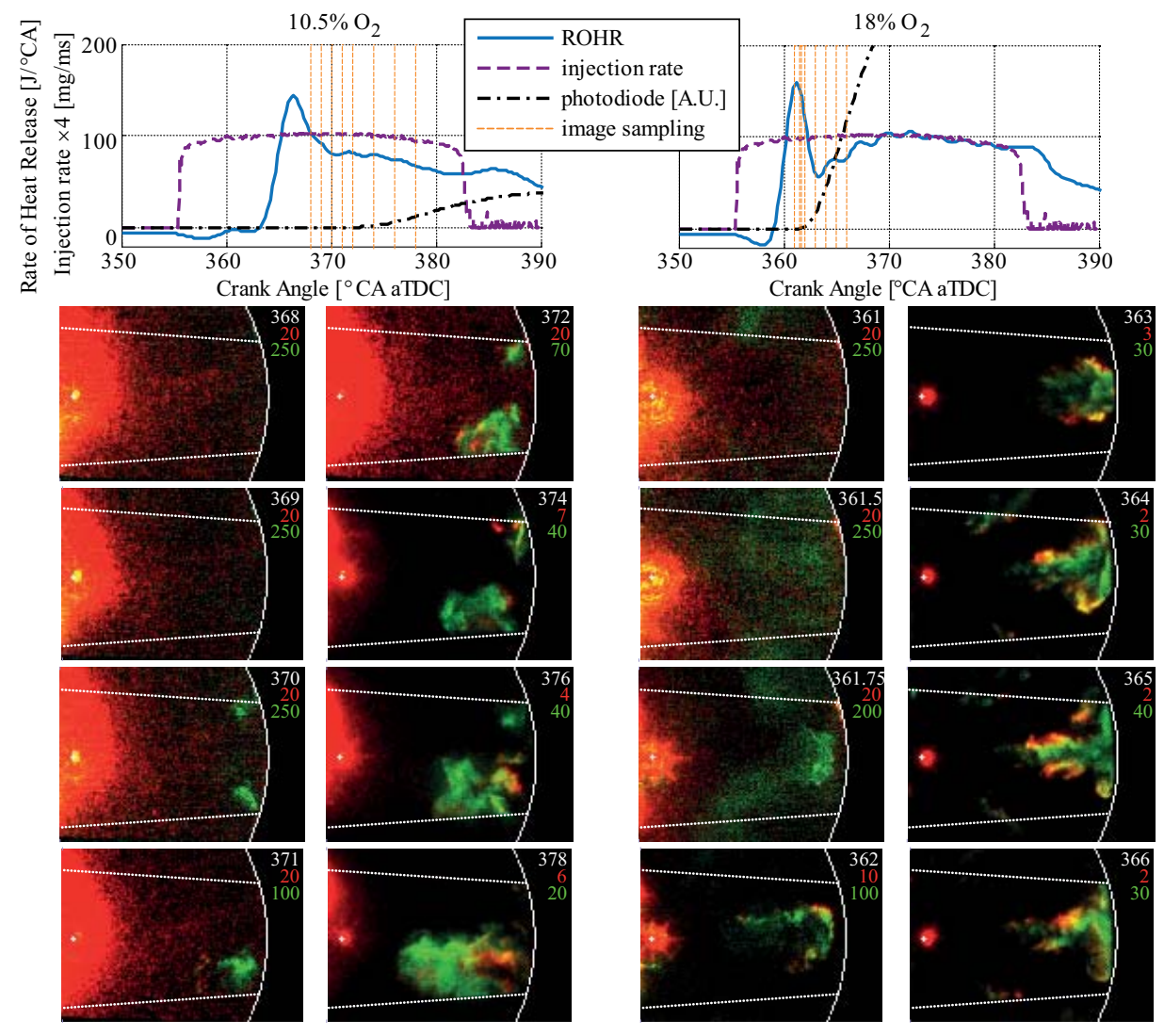

Fig. 2. Top: ROHR, injection rate and natural soot luminosity signals for $10.5 \%$ (left) and $18 \%$ (right) intake oxygen fractions. Bottom: composite single-shot images, false colored for 532-nm LIF (green, gamma=1) and LII (red, gamma $=0.8$ ) with image crank angle and color-coded signal gains are denoted in each image. (For interpretation of the references to color in this figure, the reader is referred to the electronic version of this paper.)
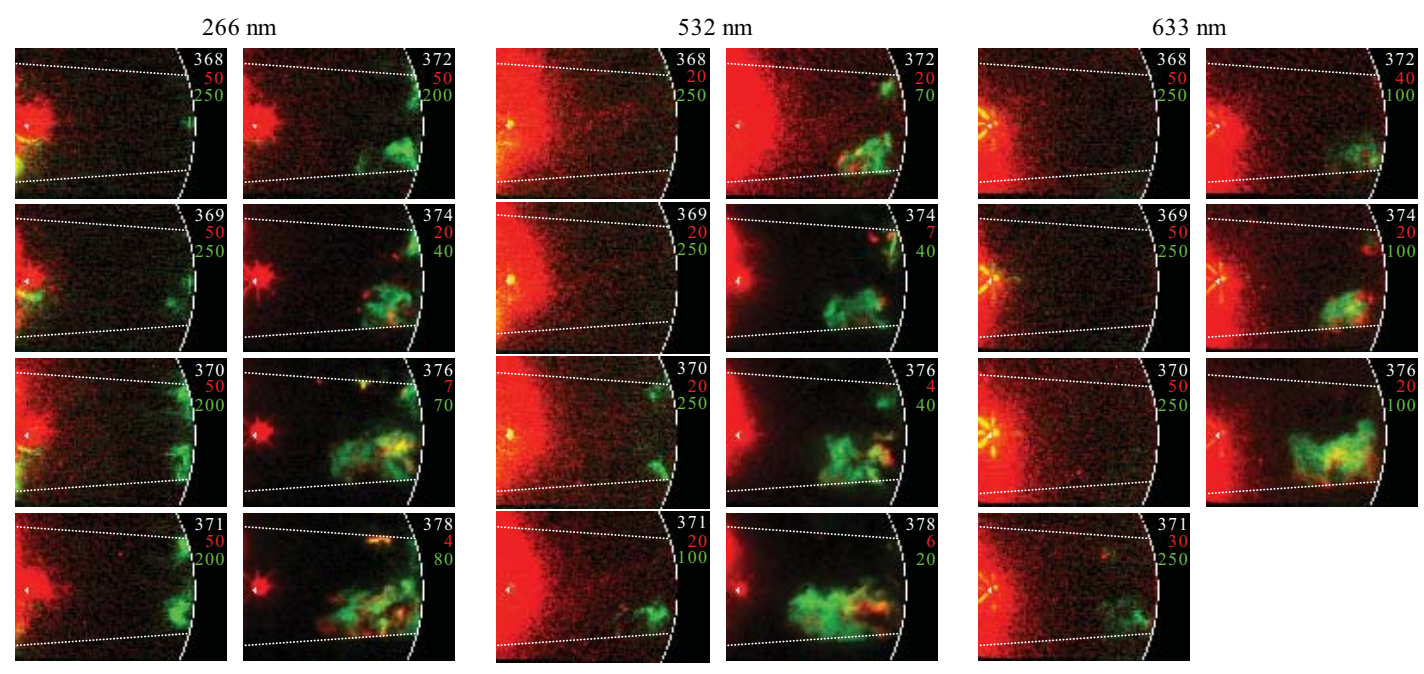

Fig. 3. Composite single-shot images, false-colored for LII excitation at $1064 \mathrm{~nm}$ (red, gamma $=0.8$ ) and LIF excitation (green, gamma=1) at 266, 532 or $633 \mathrm{~nm}$, as indicated by the column titles. Operating conditions correspond to Fig. 2. (10.5\% O2). Image crank angle and color-coded signal gains are denoted in each image. (For interpretation of the references to color in this figure, the reader is referred to the electronic version of this paper.) 


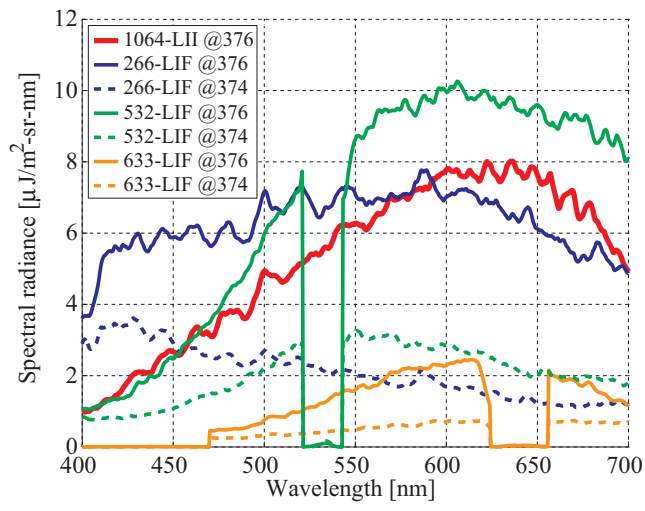

Fig. 4. Spectra of the laser-induced signal for excitation laser wavelengths of 266, 532 and 633 nm (labeled "LIF”) and 1064 nm (labeled “LII”) at 374 and 376 CAD. 10.5\% intake oxygen condition (Fig. 3).

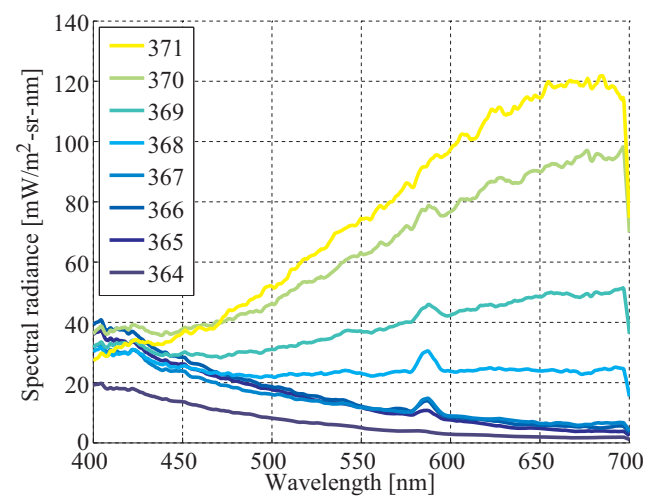

Fig. 5. Quantitative chemiluminescence emission spectra for an early crank angle range of the 10.5\% intake oxygen condition (Fig. 2, left). Legend indicates the starting crank angle of the $1{ }^{\circ} \mathrm{CA}$ camera gate. 